\title{
TUTORIAL
}

\section{Advances in architectural acoustics*}

\author{
Manfred R. Schroeder \\ Drittes Physikalisches Institut, Universität Göttingen, Germany \\ and \\ Bell Laboratories, Murray Hill, NJ 07974, U.S.A.
}

\begin{abstract}
Four areas of research in architectural acoustics in which significant advances have been achieved recently are discussed. 1. Reliable determination of subjective preference of concert hall acoustics by means of faithful (three-dimensional) reproduction of music recorded in concert halls with an acoustically realistic dummy head: The main new result, based both on real halls and digital simulations of halls, is that interaural dissimilarity should be high for high preference. This finding implies the need for more lateral diffusion in concert halls. 2. Effective methods for increasing sound diffusion: In modern wide halls with low ceilings, the two ear signals for a listener on the main floor tend to be very similar owing to strong ceiling reflections (relative to the reflections from the side walls). Thus, ceiling structures for scattering sound laterally should improve concert hall quality. Such structures, based on two number-theoretic principles (quadratic residues and primitive roots) are discussed. 3. Proper calculation of reverberation time: Sound decay rates and sound energy fluxes, based on ray theory, are given by two integral equations. Their solution, recently achieved, shows variations of decay rates by as much as $48 \%$ depending only on absorber location! (The Sabine and Eyring formulas, of course, predict no such variations and consequently lead to costly errors.) 4. Making meaningful measurements in acoustic enclosures: A recently discovered fast inverse for measurements using maximum-length pseudo-random noise is described. Some implications for making measurements with music during ongoing performance, are discussed.
\end{abstract}

PACS number: $43.55 .-\mathrm{n}$

\section{SUBJECTIVE PREFERENCE}

Acoustic quality is a subtle thing. This is true for the human voice and musical instruments and even more so for concert halls. Of course, the human ear is also quite subtle. At the threshold of hearing the hair cells in the inner ear can detect motions smaller than the diameter of a hydrogen atom. And, as we know from experience, our sense of hearing can also be very acute in perceiving minute quality differences-for example in comparing two loudspeakers, side by side, in a hi-fi salesroom.

But evaluating the acoustic quality of a concert hall is much harder. Not only does a concert hall have many more characteristics than a loudspeaker, such as direct sound, early and late sound decay rates, echoes, frequency response, variation of reverberation time with frequency, directionality ("diffusion") - all of which may differ at one location from another in the same hall-but in comparing two concert halls we are never in a position (and never will be) of listening to them side by side so to speak, alternating our attention from one to the other. We shall overlook here the singular case of two concert halls under the same roof, with doors across from each other on the same aisle, and the poor listener rotating between these two (open) doors.

\footnotetext{
* Invited lecture given at the Fall Meeting of the Acoustical Society of Japan, October 17, 1979.
} 
Yet, even if concert halls came in close-knit clusters, many listeners would still have difficulty making subtle distinctions, unless the same piece of music was played-by the same orchestra under the same conductor. (In comparing loudspeakers in the hi-fi store, we do, of course, make comparisons by listening to the same recording over different speakers.)

There is, it seems, no way out-a real dilemma! If we want to make any headway, we shall have to compromise. We can interview knowledgeable listeners, as Beranek ${ }^{1)}$ has done, hoping that a consensus of opinions will emerge. Or we can travel with an orchestra, making recordings for later comparison, as Wilkens ${ }^{2)}$ and co-workers did.

At the Drittes Physikalisches Institut, in Göttingen, we have chosen a different route. We have taken a recording of the $\mathrm{BBC}$ orchestra, playing in an anechoic environment, and reproduced it by loudspeakers positioned on the stages of numerous concert halls. ${ }^{3)}$ This assures identical music "material" in all halls, but it eliminates the important interactions between the musicians and the enclosures. These interactions must be explored separately and they are the subject of ongoing studies at Göttingen ${ }^{4)}$ and elsewhere. ${ }^{5)}$

An important prerequisite for our approach to yield meaningful results is a method of faithfully reproducing our concert hall recordings. We recorded with an acoustically refined dummy head with high-quality microphones for eardrums. But how does one transfer the recorded sound pressure signals to the eardrums of a human listener? Listening over ordinary earphones does not make the sound appear properly to come from outside the head. Listening to loudspeakers creates problems with acoustic cross-talk: The sound of each loudspeaker goes to both ears. However, this cross-talk can be eliminated by electrical (or digital) filtering ${ }^{6)}$ of the two dummy-head signals.

Suppose the complex frequency response from a loudspeaker to the near ear of a listener seated in front of two speakers in an anechoic chamber is $S(\omega)$ and that to the distant ear is $A(\omega)$. Then the Fourier transform of the signal at the right ear will be

$$
R(\omega)=S(\omega) r(\omega)+A(\omega) l(\omega),
$$

where $r(\omega)$ and $l(\omega)$ are the Fourier transforms of the right and left loudspeaker signals, respectively.
What filtered versions $\tilde{r}(\omega)$ and $\tilde{l}(\omega)$ of $r(\omega)$ and $l(\omega)$ do we have to apply to the two loudspeakers to make $R(\omega)=r(\omega)$, i.e., to make the signal at the right eardrum of the listener equal to that at the right "eardrum" of the dummy. At the same time we want to realize $L(\omega)=l(\omega)$.

The solution to this problem requires solving the following two simultaneous equations.

$$
\left.\begin{array}{l}
r(\omega)=S(\omega) \tilde{r}(\omega)+A(\omega) \tilde{l}(\omega) \\
l(\omega)=A(\omega) \tilde{r}(\omega)+S(\omega) \tilde{l}(\omega),
\end{array}\right\}
$$

yielding

and

$$
\left.\begin{array}{c}
\tilde{r}=\frac{S r-A l}{S^{2}-A^{2}} \\
\tilde{l}=\frac{S l-A r}{S^{2}-A^{2}},
\end{array}\right\}
$$

provided that $S^{2}-A^{2}$ does not vanish at any frequency of interest-a condition that has been verified experimentally. ${ }^{3)}$

Equation (3) tells us that we need two pairs of filters, one with frequency response $S /\left(S^{2}-A^{2}\right)$ to equalize the response to the near ear and another one, with response $-A /\left(S^{2}-A^{2}\right)$, to cancel the acoustic cross-talk.

Various approximations of these filters have been realized and used in evaluating the recordings made by D. Gottlob and K. F. Siebrasse in over 20 European concert halls ${ }^{3)}$ in a study supported by the Deutsche Forschungsgemeinschaft.

These recordings, reproduced in the manner just discussed, were compared - two concert halls at a time-by experienced listeners. The resulting preference scores were evaluated by multidimensional scaling. Figure 1 illustrates a typical result in the form of a so-called "preference space." Letters followed by numbers designate different concert hall seats. Arrows represent individual listeners. The meaning is as follows: Projecting the points on a listener's arrow arranges the concert hall seats in a manner reflecting that listener's overall preference. (This is not necessarily possible in two dimensions. But the data were such that the plot shown in Fig. 1 is a very close fit to the experimental preference data.) Thus, listener 7, for example, prefers hallseat E1 most, while listener 1 prefers hall-seat Q2 most.

Since all vectors (except that of listener 4) point into the right halfplane, moving the location of a 




EI - T3: 10 SEATS (4 HALLS)

1- 10: 10 DIFFERENT SUBJECTS

Fig. 1 Preference space for 10 seats in 4 concert halls with reverberation time less than $2.2 \mathrm{sec}$. Dimension D1 is called "consensus preference," and D2 reflects individual preference differences by the 10 listeners. (Measurements by D. Gottlob and $\mathrm{K}$. Siebrasse).

hall-seat in the preference space to the right (by some acoustical modification) increases the preference of that hall-seat for all listeners (except 4). Thus, ignoring listener 4 , the abscissa of the preference space (dimension D1) could be called "consensus preference." In Fig. 1, hall-seat E1 has the highest consensus preference, $\mathrm{T} 1$ the lowest.

The ordinate of the preference space (Dimension D2) reflects the listners' individual differences. Thus, while E1 is the most preferred hall-seat for listener 8 , for example, it is the least preferred for listeners 4, 6 and 3 .

What physical attributes of a hall-seat determine its location in preference space? ${ }^{6,7)}$ If we knew the answer, we might be able to build better acoustic enclosures. To answer the important question, various physical and geometrical parameters such as reverberation time and (average) width were correlated with the D1 and D2 values of each hall. The results are shown in Fig. 2 where correlations with D1 are plotted as abscissa values and correlations with $\mathrm{D} 2$ as ordinates for three physical variables: reverberation time, average hall width, and "interaural coherences."

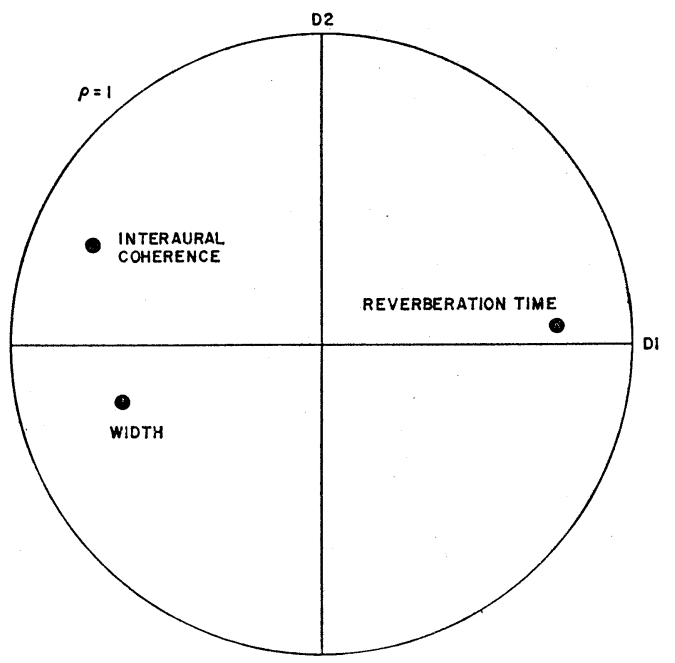

Fig. 2 Correlation of 3 objective parameters with dimensions D1 and D2 shown in Fig. 1. Interaural coherence has the highest (negative) correlation with D1 ("consensus preference").

The location of the point for reverberation time (large positive correlation with D1, small correlation with D2) means, for the hall-seats considered here, an increase in reverberation time increases consensus preference with little individual difference. This is to be expected, because the halls included in Figs. 1 and 2 have all reverberation times smaller than 2.2 secs. (Above 2.2 secs, an increase in reverberation time generally decreases musical preference for music from the classical repertoire-excluding organ music.)

The largest correlation found was between consensus preference and interaural coherence, defined as the peak value of the cross-correlation function between the two (dummy head) ear signals within a delay range of $2 \mathrm{msec}$. As can be seen in Fig. 2, the correlation with consensus preference is negative, i.e., high coherence (very similar ear signals) means low preference, and vice versa. This is one of the most significant results of the Göttingen study. It explains, among other things, why modern, wide concert halls tend to have poor acoustical acceptance: in a wide hall lateral reflections for most listeners are weak (and late arriving) compared to frontal sound from the stage and ceiling. But frontal sound leads to the highly correlated ear-signals known to be bad. This connection with a hall's width is borne out by the location of the point 
labelled "Width" in Fig. 2.

These important findings were borne out by several independent studies. ${ }^{8-10}$ )

\section{INCREASING LATERAL SOUND}

How can the sound intensity arriving laterally at a listener's ears be increased? One solution is to reflect the sound from the ceiling to the sidewalls. A particularly effective way of achieving this is to give the ceiling a surface structure according to quadratic-residue sequence ${ }^{11)}$ as shown in Fig. 3. Here the width of the "wells" is about half the shortest wavelength of the sound to be diffused and the depths $d_{n}$ vary from well to well as follows:

$$
d_{n}=\left(\lambda_{\max } / 2 N\right) \cdot\left(n^{2}\right)_{\bmod N},
$$

where $\lambda_{\max }$ is the longest wavelength to be scattered, $N$ is a prime number and $\left(n^{2}\right)_{\bmod N}$ are the residues of $n^{2}$ modulo $N$. For $N=17$, for example, one has $\left(n^{2}\right)_{\text {modil }}=0,1,4,9,16,8,2,15,13,13,15,2,8,16$, $9,4,1$, etc.

The measured reflection pattern from a scale model of the ceiling illustrated in Fig. 3 is shown in Fig. 4 for a wavelength equal to $\lambda_{\max } / 4$. One notices the desired wide pattern of reflections, a property of such ceilings for wavelengths $\lambda$ in the range

$$
\operatorname{Max}\left\{2 d, \lambda_{\max } /(N-1)\right\} \leq \lambda \leq \lambda_{\max },
$$

where $d$ is the width of the wells. ${ }^{11)}$

There are even designs that attenuate the sound that is reflected back to the main floor. They are based on "primitive root"12) sequences:

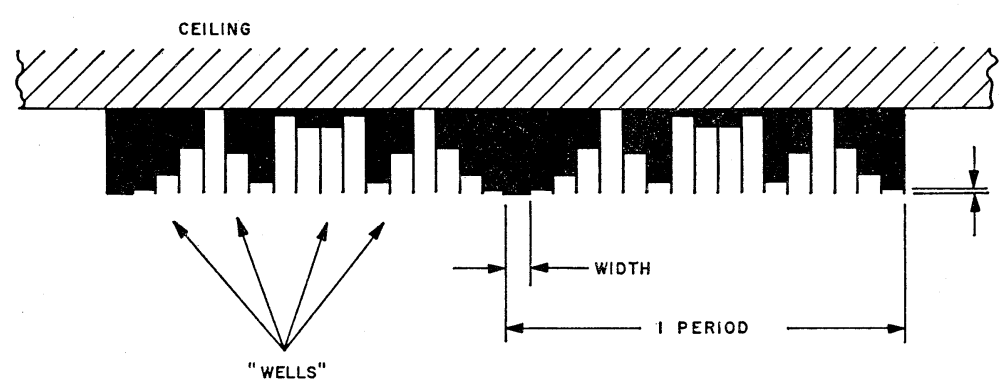

CROSS-DIMENSION OF HALL $\longrightarrow$

Fig. 3 Quadratic residue diffusor, based on quadratic residues of $N=17$, for wideband, broad sound diffusion from concert hall ceiling.

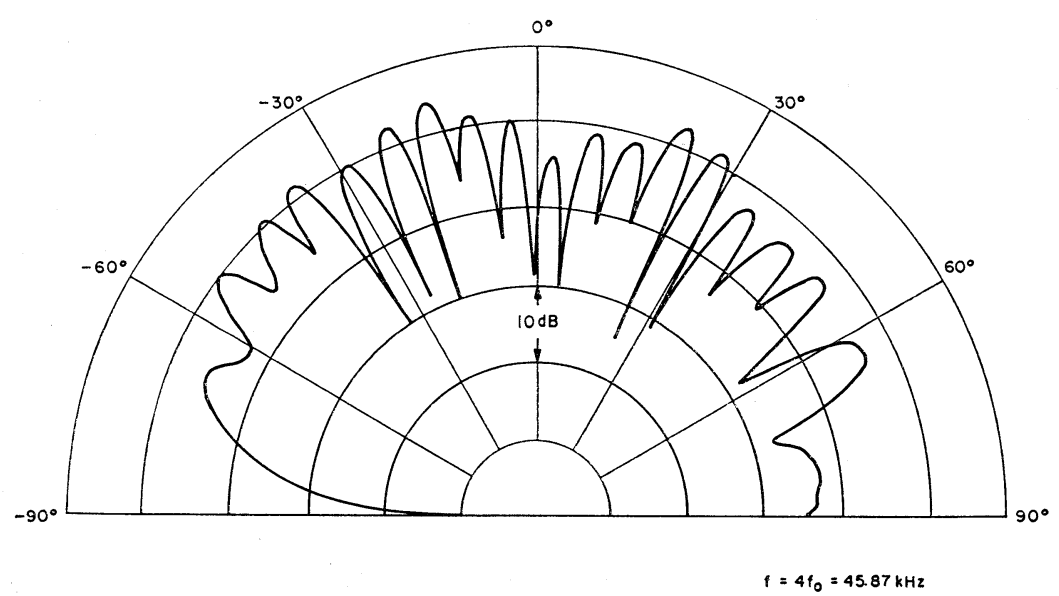

Fig. 4 Measured reflection pattern of scale model of diffusor shown in Fig. 3 at 4 times the design frequency for vertically incident sound. (Measurement by R. Gerlach and H. Henze) 


\section{R. SCHROEDER: ADVANCES IN ARCHITECTURAL ACOUSTICS}



Fig. 5 Calculated radiation pattern of primitive-root array $(N=11)$. Concentric circles are at $5 \mathrm{~dB}$ intervals. Note small "broadside" lobes at $\alpha=0^{\circ}$ and $\alpha=180^{\circ}$. This means that a reflection phase grating ("diffusor") such as shown in Fig. 3 but based on primitive roots, would have a weak specular reflection, which may be advantageous in concert hall and certain noise abatement applications.

$$
d_{n}=\left(\lambda_{\max } / 2 N\right)\left(\mathrm{g}^{n}\right)_{\bmod N},
$$

where $g$ is a primitive root of $N$. For example, for $N=11, g=2$ and $\left(g^{n}\right)_{\bmod N}=2,4,8,5,10,9,7,3,6$, 1 , etc. A radiation pattern of a linear antenna array, with $2(n-1)=20$ element spaced half a wavelength apart, and having phase angles

$$
\phi_{n}=(2 \pi / N)\left(2^{n}\right)_{\bmod 11}
$$

is shown in Fig. 5. One notices, as expected, 18 lobes of about equal magnitude and two lobes in the "broadside" directions having much lower intensity. (The spacing between two consecutive circles in Fig. 5 corresponds to 5 dB.)

If the principle of primitive-root sequences is applied to the design of "reflection phase gratings," as specified in Eq. (6), the specularly reflected sound is attenuated compared to the laterally scattered sound.

\section{THE PROPER CALCULATION OF REVERBERATION TIME}

In 1900 Wallace Clement Sabine, during his long tenure as professor in the Physics Department of
Harvard University, made extensive acoustic measurements of numerous lecture rooms and concert halls in the Boston-Cambridge area. From these measurements he distilled a formula for reverberation time. Reverberation time, he found, is inversely proportional to the average wall absorption and increases linearly with the mean-free-path of the sound rays.

Curiously, Sabine overlooked the fact, already well established in kinetic gas theory (Clausius, 1889) and integral geometry (Czuber, 1884), that the mean-free-path equals four times volume divided by surface area. Instead he hypothesized that mean-free-path varied like the third root of the volume!

Sabine's formula was, of course, only an approximation. Among other shortcomings, it did not reflect the dependence of reverberation time on the shape of the enclosure-a fact long suspected on experimental evidence and finally established beyond doubt by computer ray-tracing studies. The problem is also of interest in such (at first blush) unrelated situations as "reverberating" photons in light emitting diodes and neutron chain reactors.

As Kuttruff ${ }^{13)}$ and Joyce ${ }^{14)}$ have pointed out, the correct approach to reverberation is an integral equation for the "phonon" flux distribution over the (absorbing) surface of the enclosure. Unfortunately, the proper integral equation is a nasty one, containing, as it does, another unknown (the elusive reverberation time), thus defeating standard methods of solution.

To facilitate an iterative solution, Gilbert ${ }^{15)}$ has recently added a second integral equation. Although individually quite forbidding, the two integral equations form an ideal pair of an iterative solution. Thus, one of the most obstinate problems in architectural acoustics-that of reliably calculating reverberation time-promises finally to "go away."

In a first application of Gilbert's proposal to rectangular shapes, David Hackman, a student at Columbia University and the author, in the summer of 1979, have calculated reverberation times for 2by -4 rectangles with diffusely reflecting sides. Some of the results were astounding.

For example, by moving one of two absorbing $(\alpha=1)$ panels, covering the short side of the rectangle, to the long side of the rectangle, reverberation time was increased by as much as $48 \%$ ! Incident sound power fluxes, assumed in classical theories to 
be constant, varied by as much as $64 \%$ between different wall sections! These are drastic effects that explain the sensitivity of reverberation time to absorber location. The discrepancies with the values given by Sabine's formula differed between $+30 \%$ (!) and $-12 \%$ from the calculated resultsin spite of the fact that ideally diffuse sound reflection was assumed and the average absorption was only 0.2 or less. For the Eyring formula, discrepancies ranged from $+17 \%$ to $-21 \%$.

Much remains to be done, but the path to obtaining more reliable values of reverberation time and sound power flux distributions is clear.

\section{MEANINGFUL MEASUREMENTS}

The traditional test signals for making measurements in enclosures (and, of course, linear systems in general) have been random noise, short impulses and, occasionally, sinewaves. Much progress has been made in the generation and processing of these signals. In particular, the introduction of pseudorandom noise (based on "maximum-length" and "Legendre" sequences) for acoustic measurements has resulted in high accuracy and reproducibility and unheard of noise immunity. ${ }^{16)}$

As a very recent development, it can be reported that even fast algorithms are now known for processing time-discrete impulse responses taken with binary $( \pm 1)$ maximum-length or $m$-sequences. In matrix notations, the convolution of an $m$-sequence of period length $P=2^{k}-1$ with a periodic impulse response $h$ can be written as follows:

$$
r=M h,
$$

where $r$ and $h$ are column vectors of dimension $P$, and $M$ is a $P \times P$ cyclic matrix whose first row is one period of the $m$-sequence. $r$ is the response (output) vector. Thus the desired impulse response is

$$
h=M^{-1} r .
$$

Here $M^{-1}$ is the inverse ${ }^{17)}$ of $M$ :

$$
M^{-1}=\frac{1}{P+1}\left(M^{t}-J\right),
$$

where $M^{t}$ is the transpose of $M$ and $J$ is matrix consisting exclusively of ones.

To compute $h$ according to (9) takes $P^{2}$ multiplications and additions. With $P=2^{16}-1$ (a typical value for room-acoustical applications requiring a sampling rate of $40 \mathrm{kHz}$ and period of at least
1.6 seconds) $P^{2}=4,294,836,225$ or more than 4 billion (U.S.).

Hitherto, H. Alrutz at the Göttingen Institute has reduced the computational effort by interpolating one extra sample into the response $r$ so that its length becomes a power of 2. Thus, the (fast) Fourier transform of $r$ can be obtained by about $(P+1) \log _{2}(P+1)$, i.e., several thousand times fewer, operations. Division by the Fourier transform of the $m$-sequence and another Fourier transformation then yields a good approximation to $h$.

But there is now a direct fast method to evaluate $M^{-1}$. It is based on the fact that bordering $M$ by one row and one column of ones results in a Hadamard matrix of order $2^{k}$ which in turn (after some reordering of rows and columns) can be written as a Kronecker product involving only the Hadamard matrix of order 2 :

$$
H_{2}=\left(\begin{array}{rr}
1 & 1 \\
1 & -1
\end{array}\right)
$$

and identity matrices. ${ }^{17)}$ (The fact that fast Fourier transforms are possible is exactly the same: the discrete Fourier transform matrix can be written as a Kronecker product involving only the order- 2 Fourier matrix and certain permutation matrices and "twiddle factors.")

In spite of these successes, room-acoustical evaluations in the future should rely on measurements using the music as performed on the stage of a concert hall as the test signal. After all, the music he hears is all the human listener can go by to judge acoustical quality. Reverberation time, especially the subjectively important early reverberation time, should be measurable by measuring autocorrelation functions both near the musical instruments (on the stage) and near a listener's (or dummy's) ear and comparing the two. The important interaural coherence should be made measurable by cross-correlating the output signals from a dummy (or an actual human head fitted with probe microphones near his eardrums).

Progress is thereby being made on another urgent problem: that of making measurements in occupied halls, in the presence of an audience. Ultimately, it should be possible to determine-not just reverberation time-but everything that is important to the human listener from measurements of sound 


\section{R. SCHROEDER: ADVANCES IN ARCHITECTURAL ACOUSTICS}

pressure near his ears during a performance. The human listener doesn't need bangs, pops and hisses to judge a hall's quality. All he needs is the music. But he has a complicated brain between his ears and it remains to be seen whether we can really duplicate his acoustic preference judgment process by a machine.

\section{REFERENCES}

1) L. L. Beranek, Music, Acoustics and Architecture, (Wiley, New York, 1962).

2) H. Wilkens, "A Multidimensional Description of Subjective Judgment of Concert-hall Acoustics," Acustica. 38, 10-23 (1977).

3) M. R. Schroeder, D. Gottlob, and K. F. Siebrasse, "Comparative Study of European Concert Halls," J. Acoust. Soc. Am. 56, 1195 (1974).

4) A. H. Marshall, D. Gottlob, and H. Alrutz, "Acoustical Auditions Preferred for Ensemble," J. Acoust. Soc. Am. 64, 1437 (1978).

5) H. Wilkens and B. Koterba, "A Comparison of the Evaluation of Different Room Acoustical Situations When the Sound Sources Are either Loudspeakers or an Orchestra," Acustica 40, 291297 (1978).

6) T. Yamamoto and F. Suzuki, "Multivariate Analysis of Subjective Measures for Sound in Rooms and the Physical Values of Room Acoustics," J. Acoust. Soc. Jpn. 32, 599-605 (1976).

7) K. Yamaguchi, "Multivariate Analysis of Subjective and Physical Measures of Hall Acoustics," J. Acoust. Soc. Am. 54, 1271-1279 (1972).

8) Y. Ando, "Subjective Preference in Relation to Objective Parameters of Music Sound Fields with a Single Echo," J. Acoust. Soc. Am. 62, 14361441 (1977).

9) D. Gottlob, A. Kohlrausch, and M. R. Schroeder, "Einfluß raumakustischer Parameter auf die subjektive Beurteilung von Schallfeldern," Fortschr. Akustik, DAGA '78, Bochum (1978).

10) Y. Ando and D. Gottlob, "Effects of Early Multiple Reflections on Subjective Preference Judgments of Music Sound Fields," J. Acoust. Soc. Am. 65, 524 (1979).

11) M. R. Schroeder, "Binaural Dissimilarity and Optimum Ceilings for Concert Halls: More Lateral Sound Diffusion," J. Acoust. Soc. Am. 65, 958-963 (1979).

12) M. R. Schroeder, "Constant-Amplitude Antenna Arrays with Beam Patterns Whose Lobes Have Equal Magnitudes," to be published in Archiv für Elektronik und Übertragungstechnik (AEÜ).

13) H. Kuttruff, "Simulierte Nachhallkurven in Rechteckräumen mit diffusem Schallfeld," Acustica. 25, 333-342 (1971).

14) W. B. Joyce, "Exact Effect of Surface Roughness on the Reverberation Time of a Uniformly Absorbing Spherical Enclosure," J. Acoust. Soc. Am. 64, 1429-1436 (1978).

15) E. N. Gilbert, "An Iterative Calculation of Auditorium Reverberation," to be submitted to J. Acoust. Soc. Am..

16) M. R. Schroeder, "Integrated-impulse Method Measuring Sound Decay without Using Impulses," J. Acoust. Soc. Am. 66, 497-500 (1979).

17) M. Harwit and N. J. A. Sloane, Hadamard Transform Optics, (Academic Press, New York, 1979), pp. 208-209. 